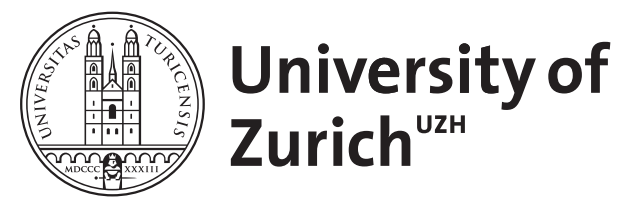

\title{
Eye movements reveal mental looking through time
}

\author{
Stocker, Kurt ; Hartmann, Matthias ; Martarelli, Corinna S ; Mast, Fred W
}

\begin{abstract}
People often make use of a spatial "mental time line" to represent events in time. We investigated whether the eyes follow such a mental time line during online language comprehension of sentences that refer to the past, present, and future. Participants' eye movements were measured on a blank screen while they listened to these sentences. Saccade direction revealed that the future is mapped higher up in space than the past. Moreover, fewer saccades were made when two events are simultaneously taking place at the present moment compared to two events that are happening in different points in time. This is the first evidence that oculomotor correlates reflect mental looking along an abstract invisible time line during online language comprehension about time. Our results support the idea that observing eye movements is likely to "detect" invisible spatial scaffoldings which are involved in cognitively processing abstract meaning, even when the abstract meaning lacks an explicit spatial correlate. Theoretical implications of these findings are discussed.
\end{abstract}

DOI: https://doi.org/10.1111/cogs.12301

Posted at the Zurich Open Repository and Archive, University of Zurich

ZORA URL: https://doi.org/10.5167/uzh-119537

Journal Article

Accepted Version

Originally published at:

Stocker, Kurt; Hartmann, Matthias; Martarelli, Corinna S; Mast, Fred W (2016). Eye movements reveal mental looking through time. Cognitive Science, 40(7):1648-1670.

DOI: https://doi.org/10.1111/cogs.12301 


\title{
Eye movements reveal mental looking through time
}

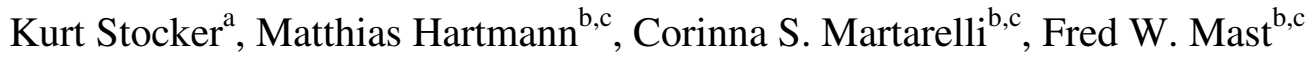 \\ ${ }^{a}$ Department of Psychology, University of Zurich, Binzmühlestrasse 14/25, Zurich, \\ Switzerland \\ ${ }^{\mathrm{b}}$ Department of Psychology, University of Bern, Fabrikstrasse 8, 3012 Bern, Switzerland \\ ${ }^{c}$ Center for Cognition, Learning and Memory, University of Bern, Fabrikstrasse 8, 3012 \\ Bern, Switzerland
}

Keywords: Eye movements; Language comprehension; Mental time line; Time; Space; Mental simulation; Metaphor; Embodiment

\begin{abstract}
People often make use of a spatial "mental time line" to represent events in time. We investigated whether the eyes follow such a mental time line during online language comprehension of sentences that refer to the past, present, and future. Participants' eye movements were measured on a blank screen while they listened to these sentences. Saccade direction revealed that the future is mapped higher up in space than the past. Moreover, fewer saccades were made when two events are simultaneously taking place at the present moment compared to two events that are happening in different points in time. This is the first evidence that oculomotor correlates reflect mental looking along an abstract invisible time line during online language comprehension about time. Our results support the idea that observing eye movements is likely to "detect" invisible spatial scaffoldings which are involved in cognitively processing abstract meaning, even when the abstract meaning lacks an explicit spatial correlate. Theoretical implications of these findings are discussed.
\end{abstract}




\section{Introduction}

Eyes can be a window into the mind. They for instance reveal insights into the spatiotemporal characteristics of ongoing cognitive processes, such as language processing or mental imagery (e.g., Altmann, 2004; Grant and Spivey, 2003; Hartmann, Martarelli, Mast, \& Stocker, 2014; Huette, Winter, Matlock, Ardell, \& Spivey, 2014; Johansson and Johansson, 2014; Spivey and Geng, 2001; Van Gompel, Fischer, Murray, \& Hill, 2007). A widespread technique for studying online language comprehension with eye movements is the so-called visual world paradigm (Carreiras \& Clifton, Jr., 2004; Cooper, 1974; Huettig, Rommers, \& Meyer, 2011; Tanenhaus, Spivey-Knowlton, Eberhard, \& Sedivy, 1995). In the visual world paradigm, participants are looking at a visual scene and at the same time are listening to an utterance which relates to the visual scene. This paradigm has revealed that language comprehension directly corresponds to the exploration of the visual world. For example, participants fixate their eyes on a depicted lion upon hearing part or all of the word 'lion', or they fixate their eyes on a lion, zebra, and a snake upon hearing the word 'Africa' (Cooper, 1974). More recently, the visual world paradigm has also been used to investigate eye movements during online language comprehension of dynamic thought. For instance, the mental simulation of the speed of an entity going through space (e.g., a lion is ambling vs. dashing toward on object) is also reflected in eye movements (Lindsay, Scheepers, \& Kamide, 2013; Speed \& Vigliocco, 2014). Furthermore, eye movements have been found to reflect an abstract kind of motion called fictive motion. Fictive motion is the description of a static scene with motion words (Talmy, 1996). It has for example been found that the eyes inspect the same visual object (e.g., an image of a road) during a fictive motion description (e.g., 'The road goes through the desert') longer when the road terrain was first described as rough ('The desert is hilly') as compared to smooth ('The desert is flat') (Richardson \& Matlock, 2007; cf. also Mishra \& Singh, 2010).

It is also a well-established phenomenon that the spatial properties of absent visual 
percepts can influence eye movements, i.e., when these percepts are only imagined or recalled (e.g., Beech, 1980; Brandt \& Stark, 1997; Borst \& Kosslyn, 2008; Laeng \& Teodorescu, 2002; Martarelli \& Mast, 2011). In the context of online eye-tracking investigations of spoken language comprehension, this phenomenon has been studied by using a paradigm that is closely related to the visual world paradigm and is sometimes referred to as the blank screen paradigm (Altmann, 2004; Huette et al., 2014; Johansson, Holsanova, \& Holmqvist, 2006; Mishra \& Singh, 2010; Spivey \& Geng, 2001). In this paradigm, participants' eye movements on a blank screen are recorded while they listen to content that in principle is perceptible and can thus be mentally projected onto the screen. In one of the first studies using this paradigm (Spivey \& Geng, 2001; cf. also Richardson \& Spivey, 2000), it has been shown that listening to a description of the lower floors of a tall building, followed by the description of the higher floors, leads to corresponding upward eye movements on the blank screen. As Spivey and Geng wrote: "...[the] construction of a mental image is almost 'acted out' by the eye movements, and a mental search of internal memory is accompanied by an oculomotor search of external space" (p. 235).

More recently__ as with the visual world paradigm_— the blank screen paradigm has also been used to investigate eye movements during online language comprehension of more abstract thought. For instance, in a recent eye-tracking study, Huette and colleagues (2014) found that listening to expressions that emphasize the ongoing nature of an action (e.g., he was climbing; past progressive) evokes a wider distribution of eye fixations on the blank screen than expressions emphasizing the end point of an action (e.g., he climbed; simple past). Thus, abstract linguistic differences can entail specific oculomotor behavior.

To the best of our knowledge, all language comprehension studies that investigated eye movements searched for oculomotor correlates which relate to objects in space (where the "object" can either be animate or inanimate). Thus, as mentioned, it has for instance been investigated whether eye movements reflect the speed of an object going through space 
(Lindsay et al., 2013; Speed \& Vigliocco, 2014) or if they reflect looking along a static object (Richardson \& Matlock, 2007). While we in the current study also used sentences that refer to objects in space (e.g, "Now I'm watching TV. Before that I was listening to music"; cf. below), we did not investigate spatial correlates of these objects_— but rather focused on abstract (non-perceivable) temporal correlates (e.g., of "before"). Thus, we address the question whether the abstract concept of time itself is systematically reflected in eye movements during language processing. Time is abstract in the sense that the passing of time, or the representation of different events in time, has no obvious perceptual correlate (one does not see a timeline when processing temporal information). Nevertheless, recent evidence has shown that time is processed within invisible spatial scaffoldings. Particularly, the flow of time is conceptualized as progressing along a mental time line (Santiago, Lupáñez, Pérez, \& Funes, 2007; Torralbo, Santiago, \& Lupiañez, 2006; Ulrich \& Maienborn, 2010; Ulrich et al., 2012), in analogy to number magnitudes that are conceptualized as progressing along a mental number line (e.g., Dehaene, Bossini, \& Giraux, 1993; Fischer, Castel, Dodd, \& Pratt, 2003; Hartmann, Grabherr, \& Mast, 2012; Hubbard, Piazza, Pinel, \& Dehaene, 2005; Lourenco \& Longo, 2010). The question whether the eyes follow such spatial scaffoldings for abstract thought has hardly been investigated yet. In one of the few studies addressing this issue, it has been shown that the eyes follow a leftward/downward-small and a rightward/upward-large spatial-numerical association when speaking out a random sequence of numbers (Loetscher, Bockisch, Nicholls, \& Brugger, 2010), or partially also during mental addition and subtraction (Hartmann, Mast, \& Fischer, 2015). With respect to the spatialtemporal association, we recently showed that the eyes shifted rightward and upward when thinking about the future compared to the past (Hartmann et al., 2014). While these studies revealed that the eyes follow an abstract mental number line or time line for internally generated stimuli (e.g., numbers, future and past thoughts), the present study is to our knowledge the first that investigates if the eyes follow an abstract mental-time-line 
scaffolding during language comprehension.

We investigated whether the eyes follow such a mental time line during online language comprehension of sentences that refer to the past, present, and future. Sentences with before referred to the past, sentences with after to the future, and sentences with sametime to the present moment (e.g., 'Now I'm watching TV. Before that I was listening to music'; 'Now I'm watching TV. After that I will be listening to music'; 'Now I'm doing my homework. At the same time I'm listening to music').

Processing these temporal relations could be spatially reflected in different ways through eye movements__ a possibility, which we will now elaborate on in more detail. In Western culture the future is ahead and the past behind (Clark, 1973; Lakoff \& Johnson, 1980b), suggesting that time is mentally construed to flow along a back-to-front time line (Hartmann \& Mast, 2012; Miles, Nind, \& Macrae, 2010; Torralbo et al., 2006; Ulrich et al., 2012), often also referred to as a "sagittal" time line. As a further time-line option in Western culture, the past is to one's left and the future to one's right (Bergen \& Chan Lau, 2012; Casasanto \& Jasmin, 2012; Cooperrider \& Núñez, 2009; Santiago et al., 2007; Sinha, Sinha, Zinken, \& Sampaio, 2011; Torralbo et al., 2006; Tversky, Kugelmass, \& Winter, 1991; Ulrich \& Maienborn, 2010). While some other cultures show different time spatialization patterns (Bergen \& Chan Lau, 2012; Boroditsky, 2001; Boroditsky \& Gaby, 2010; Le Guen, 2012; Le Guen \& Pool Balam, 2012; Núñez \& Sweetser, 2006; Sinha et al., 2011; Tversky et al., 1991), thus far no culture or language has been found where time is not spatialized in a systematic way (Stocker, 2014b). The methodological approaches supporting the existence of a specifically directed (e.g., behind-to-ahead, left-to-right, etc.) mental time line include: linguistic analysis of spoken-language temporal expressions (Clark, 1973; Lakoff \& Johnson, 1980b; Lakoff \& Johnson, 1999; Stocker, 2012; Stocker, 2014a), for instance English expressions like "Trouble lies ahead" or "The worst of it is behind us" (Clark, 1973, p. 51); linguistic analysis of signed-language temporal expressions (Boyes Braem, 1992; Brennan, 
1983; Klima \& Bellugi, 1979), for instance signs in British Sign Language (and other sign languages) that locate the past just above the right shoulder and the future further ahead from this point (Brennan, 1983, p. 12), or signs in British Sign Language in front of the signer's body that spatialize duration as extending from left to right (Brennan, 1983, p. 19); cospeech gestural analysis of spoken-language temporal expressions (Casasanto \& Jasmin, 2012; Cooperrider \& Núñez, 2009; Núñez, Cooperrider, Doan, \& Wassmann, 2012; Le Guen, 2012), for instance English co-speech gestures that locate the past further behind (or further to the left) and the future further ahead (or further to the right) (Casasanto \& Jasmin, 2012; Cooperrider \& Núñez, 2009); analysis of graphic output of temporal order (Bergen \& Chan Lau, 2012; Boroditsky \& Gaby, 2010; Le Guen \& Pool Balam, 2012; Sinha et al., 2011; Tversky et al., 1991), for instance cards (say, tadpole, froglet, frog) laid out from left to right by native American English speakers (Bergen \& Chan Lau, 2012); observing or evoking body movement along the sagittal axis while processing time (Hartmann \& Mast, 2012; Miles, Nind, et al., 2010); and using two-alternative-forced-choice temporal classifications (e.g., past-left/future-right) paradigms (Boroditsky, 2001; Casasanto \& Bottini, 2013; Hartmann \& Mast, 2012; Miles, Betka, Pendry, \& Macrae, 2010; Miles, Tan, Noble, Lumsden, \& Macrae, 2011; Santiago et al., 2007; Torralbo et al., 2006; Ulrich et al., 2012; Ulrich \& Maienborn, 2010).

Given that our participants were from a Western culture (native speakers of German), the eyes might follow a sagittal back-to-front or transversal left-to-right mental time line while processing language about time. However, while analysis of eye movements on the screen can be used for assessing the transversal (left-right) and vertical (up-down) projection of temporal progression onto space, it offers no direct method for assessing the sagittal axis. Nonetheless, it is possible to mentally project a sagittal axis as extending "into the screen" (e.g., Kaschak et al., 2005). Moreover, we know from visual perception that objects lying sagittally ahead of an observer are perceived as higher with increasing distance from the 
observer, even when in physical reality the objects are not higher, but only further away (Ooi, Wu, \& He, 2001; Yang \& Purves, 2003). Thus, if participants were able to mentally project the future as extending sagittally out of their body, then the geometrical projection onto screen coordinates would lead to future locations higher on the screen than past locations. In other words, the mental time line going away from us might be "ahead" and "up" at the same time. To test the up/down and left/right predictions, we analyzed vertical and horizontal direction of saccades during temporal processing. If ocular processing is influenced by spatiotemporal associations, different amounts of horizontal and vertical saccades are expected for the processing of the different temporal relations.

As a complementary approach to test our hypothesis that we mentally simulate looking along the time line during language comprehension about time, we also analyzed the total number of saccades and the fixation patterns for each of the given temporal relations. If we mentally look along the mental time line, then processing two sequential (temporally separated) events (before, after) should lead to more changes in spatial orientation (i.e., saccades) than processing two concurrent (temporally non-separated) events (same-time) because two temporally separated events would be represented at two distinct locations on the time line.

\section{Methods}

\subsection{Participants}

Sixteen right-handed undergraduate students from the University of Bern participated in this study for course credit (10 women, mean age: 21.8 , range: $19-27$ years). The study was approved by the local Ethics Committee, and participants gave informed consent prior to the study.

\subsection{Stimuli}


We used sentence pairs where the second sentence referred to the past, present, or future. The first sentence described an event that occurs in the present and always started with 'now.' The second sentence described an event that either occurs prior to (before), subsequent to (after), or simultaneous (same-time) with the first event. We first created an initial set of 30 such sentence pairs, 10 for each category (before, after, same-time).

Sentences were recorded with a hypothesis-blind German speaker in the phonetics laboratory of the University of Zurich. To maintain the natural temporal structure of language, the duration of the sentences was not changed artificially. The mean length of the sentence pairs was $4088 \mathrm{~ms}$, and the mean onset of the word expressing the temporal relation (before, after, same-time) was $2335 \mathrm{~ms}$. Given that the word at the same time ("gleichzeitig" in German) is slightly longer than the words before ("vorher") and after ("nachher"), the mean total duration of the same-time sentences was slightly longer (307 ms). However, the duration between the offset of the temporal-relation word and the end of the sentence did not differ between the categories $(F<1)$.

Two sets of stimuli were created in total. One set contained the 30 sentences created initially. In the other set, the contents of the before and after sentences were exchanged (e.g., 'Now I'm watching TV. Before that I was listening to music' was changed to 'Now I'm watching TV. After that I will be listening to music'). In addition, the content of the same-time sentences were exchanged (e.g., 'Now I'm listening to music. At the same time I'm doing my homework' was changed to 'Now I'm doing my homework. At the same time I'm listening to music' $).{ }^{1}$ Counterbalancing the contents of the sentences should minimize time-unspecific effects. An independent sample $(N=12)$ rated each two-sentence pair with respect to arousal and to how dynamic the content of the sentence pair was perceived on a seven-point Likert

\footnotetext{
${ }^{1}$ Out of the 30 sentences 15 were phrased with the first person singular ('Now I ...') and 15 with the third person singular ('Now he/she ...'). Thus, the person perspective was varied in the experiment. However, given that we had no hypothesis in relation to this distinction and only a limited number of repetitions of this factor, we did not analyze our data with respect to the person perspective.
} 
scale. No differences between the before, after, and same-time sentences were found $(F<1)$.

To disguise the purpose of this study, we used 40 other sentences as distractors (e.g., 'The book store is already closed, but the salesman is still there').

\subsection{Eye movement recording}

Eye movements were recorded using an SMI RED tracking system (SensoMotoric Instruments, Teltow, Germany). Data were registered at a sampling rate of $50 \mathrm{~Hz}$, a spatial resolution of $0.1^{\circ}$, and a gaze position accuracy of $0.5^{\circ}$. The primary outcome of the eye tracking device are fixations, defined by a minimum fixation duration of $80 \mathrm{~ms}$ and a dispersion of 100 pixels. ${ }^{2}$ The eye tracker we used does not allow for detecting saccades directly (which would be based on velocity criterion and requires a higher sampling rate). Nevertheless, to assess the relative change in spatial attention induced by the temporal content, our main analyses were based on saccades, determined by subtracting fixations and blink events from the original gaze stream using Be-Gaze software (SensoMotoric Instruments, Teltow, Germany). The stimuli were presented on a 17 -inch screen using Experiment Center Software, and eye data were recorded with I-View X Software (both developed by SensoMotoric Instruments).

\subsection{Procedure}

Participants were seated in front of the computer screen and asked to carefully listen to a set of sentences including 10 before, 10 after, 10 same-time, and 40 distractor sentences while looking at an empty (gray) screen (within-subjects design). The 70 sentences/sentence pairs were presented via loudspeakers in random order with a delay between the onset of one

\footnotetext{
${ }^{2}$ The algorithm checks the dispersion of consecutive data points in a moving window by summing the differences between the points' maximum and minimum $\mathrm{x}$ and $\mathrm{y}$ values $([\max (\mathrm{x})-\min (\mathrm{x})]+[\max (\mathrm{y})-\min (\mathrm{y})])$. If the sum is below 100 pixels, the window represents a fixation and expands until the sum exceeds 100 pixels. The final window is registered as a fixation at the centroid of the window points with the given onset time and duration.
} 
sentence pair to the onset of the next sentence pair of $6 \mathrm{~s}$. To encourage semantic processing of the contents, participants were told that from time to time questions about the content of some sentences would be asked. In fact, 10 statements about the content of a previously presented sentence appeared on the screen during the experiment. We randomly selected 10 of the 40 distractor sentences and generated a question for each of these sentences (requiring 5 true and 5 false responses). These questions always followed the corresponding sentences. Moreover, we directed each participant's attention away from eye movements by means of a cover story. Participants were told that this study was about the relationship between pupil size and cognitive processes during sentence comprehension. Participants were asked to listen carefully to the sentences and if statements about the contents of previously presented sentences appeared on the screen, to indicate as quickly and accurately as possible whether the statement was true or false by saying yes or no. They were told that they are free to move their eyes, but they should keep looking at the screen. After the experiment and before debriefing, participants were asked to guess the hypothesis of this experiment. The responses to the statements and guessed hypotheses were noted by the experimenter.

\subsection{Data Analysis}

We were interested in what impact the words expressing the different temporal relations (before, after, same-time) had on the direction of saccades (upward vs. downward, leftward vs. rightward) and on the total number of saccades (e.g., is the processing of different temporal relations correlated with more eye movements?). We analyzed saccades that were performed in the time interval between 500 and $3500 \mathrm{~ms}$ after the onset of the word expressing the temporal relation. This interval was chosen because the duration of the word expressing the temporal relation was at least $500 \mathrm{~ms}$ and we expected no semantic processing of the temporal relation before then, and $3500 \mathrm{~ms}$ include the whole sentence in every trial. We excluded 72 saccades $(2.7 \%)$ from the analysis because they were outside the screen. 
Each saccade was categorized according to its vertical (upward vs. downward) and horizontal (leftward vs. rightward) displacement in space. For example, a saccade categorized as upward and rightward means that the $\mathrm{y}$-coordinate of the saccade changed in an upward, and the $\mathrm{x}$ coordinate in a rightward direction from the start to the stop of the saccade (whereby the relative magnitudes of the $\mathrm{x}$ and $\mathrm{y}$ displacement was irrelevant for this classification). We for each participant computed the proportion of upward saccades, the proportion of rightward saccades, and the sum of saccades over all trials for each temporal category. A proportion of upward saccades of $65 \%$ for instance means that $65 \%$ of all saccades were directed upward, and consequently $35 \%$ were directed downward. To capture temporal aspects of the impact of the word expressing the temporal relation, we computed these values separately for $1 \mathrm{~s}$ time intervals and created the variable time interval $(1=$ saccades that were initiated in the time window between 500 and $1500 \mathrm{~ms}$ after the onset of the critical time word; $2=$ saccades between 1500 and $2500 \mathrm{~ms} ; 3=$ saccades between 2500 and $3500 \mathrm{~ms}$ ). We illustrate what parts of the sentences occur within these time intervals with the following three examples: “Now I'm reading a book_—at the same time I'm ruffling a cat; Now I'm watching TV_— after(wards) I'm listening to music; Now I'm on the boat—-before I was on an island." The parts "I'm ruffling," "I'm listening," and "I was on an" are captured by Interval 1. The remaining word(s) are captured by Interval 2, and 1s silence is captured by Interval 3. The allocation of contents to time intervals was similar across all sentences.

Three separate repeated measures ANOVAs with the variables temporal relation (before, after, same-time) and interval $(1,2,3)$ were computed for the proportion of upward saccades, the proportion of rightward saccades, and the total number of saccades. Paired $t$ tests were applied for post-hoc tests. For the proportion of upward and rightward saccades, we also performed planned comparisons between before and after sentences (using paired $t$ tests; uncorrected $p$-values are reported). 


\section{Results}

All participants gave correct responses for at least eight out of the 10 statements, showing that the sentences were processed on a semantic level. Moreover, no participant correctly guessed our hypothesis.

For the mean proportion of upward saccades, the ANOVA revealed a trend for temporal relation $(p=.083)$. Most importantly, the planned comparison showed that significantly more upward saccades were made when listening to after-sentences than when listening to before-sentences $(56.8 \%$ vs. $50.5 \% ; p=.004)$. The mean proportion of upward saccades for each time and interval is presented in Figure 1a. Upward saccades while listening to after-sentences were above chance level $(56.8 \%, S D=6.9 \%, t(15)=3.94, p=$ $.001)$, and also same-time sentences differed from chance $(54.5 \%, S D=7.3 \%, t(15)=2.49, p$ $=.025)$, whereas upward saccades while listening to before-sentences turned out to be nonsignificantly different from chance level $(p=.76)$.

For the proportion of rightward saccades, the ANOVA revealed no significant effects (for all $p>.245$, see Table 1), and the planned comparison confirmed that there was no difference between before and after sentences $(p=.662)$. The mean proportion of rightward saccades for each time and interval is presented in Figure 1b. Only the proportion of rightward saccades for same-time sentences $(M=53.4, S D=5.2)$ differed significantly from chance, $t(15)=2.59, p=.021$. This effect might reflect that there are asymmetries in the proportion of rightward saccades even in a "baseline", suggesting that $50 \%$ might not be an appropriate reference. We therefore do not interpret this effect further and focus on the comparison between the three categories (which is the relevant comparison for the purpose of this study).

For the total number of saccades, the ANOVA revealed a significant main effect of interval and a significant interaction between temporal relation and interval (see Table 1). 
Post-hoc tests revealed that the total number of saccades was higher in Interval $2(M=19.4)$ when compared with Interval $1(M=17.8, p=.045)$ and Interval $3(M=17.9, p=.016)$.

Interestingly, in Interval 3, fewer saccades were made when listening to same-time than when listening to before (15.9 vs. $19.4 ; p=.008)$ and after sentences (15.9 vs. $18.3 ; p=.025$; see Figure 1c).

Table 1

Results of the Analysis of Variance with the Variables Temporal relation (TR) and Interval (I)

\begin{tabular}{|c|c|c|c|c|c|c|c|c|c|}
\hline & \multicolumn{3}{|c|}{$\begin{array}{l}\text { Proportion of } \\
\text { upward saccades }\end{array}$} & \multicolumn{3}{|c|}{$\begin{array}{l}\text { Proportion of } \\
\text { rightward saccades }\end{array}$} & \multicolumn{3}{|c|}{$\begin{array}{l}\text { Total number } \\
\text { of saccades }\end{array}$} \\
\hline & $F$ & $p$ & $\eta_{p}^{2}$ & $F$ & $p$ & $\eta_{\mathrm{p}}^{2}$ & $F$ & $p$ & $\eta_{p}^{2}$ \\
\hline TR & 2.72 & .083 & .15 & 0.30 & .740 & .02 & 0.86 & .433 & .05 \\
\hline I & 0.23 & .796 & .02 & 0.06 & .939 & $<.01$ & 3.82 & .033 & .20 \\
\hline TR $x$ I & 0.50 & .737 & .03 & 1.40 & .245 & .09 & 3.08 & .022 & .17 \\
\hline
\end{tabular}



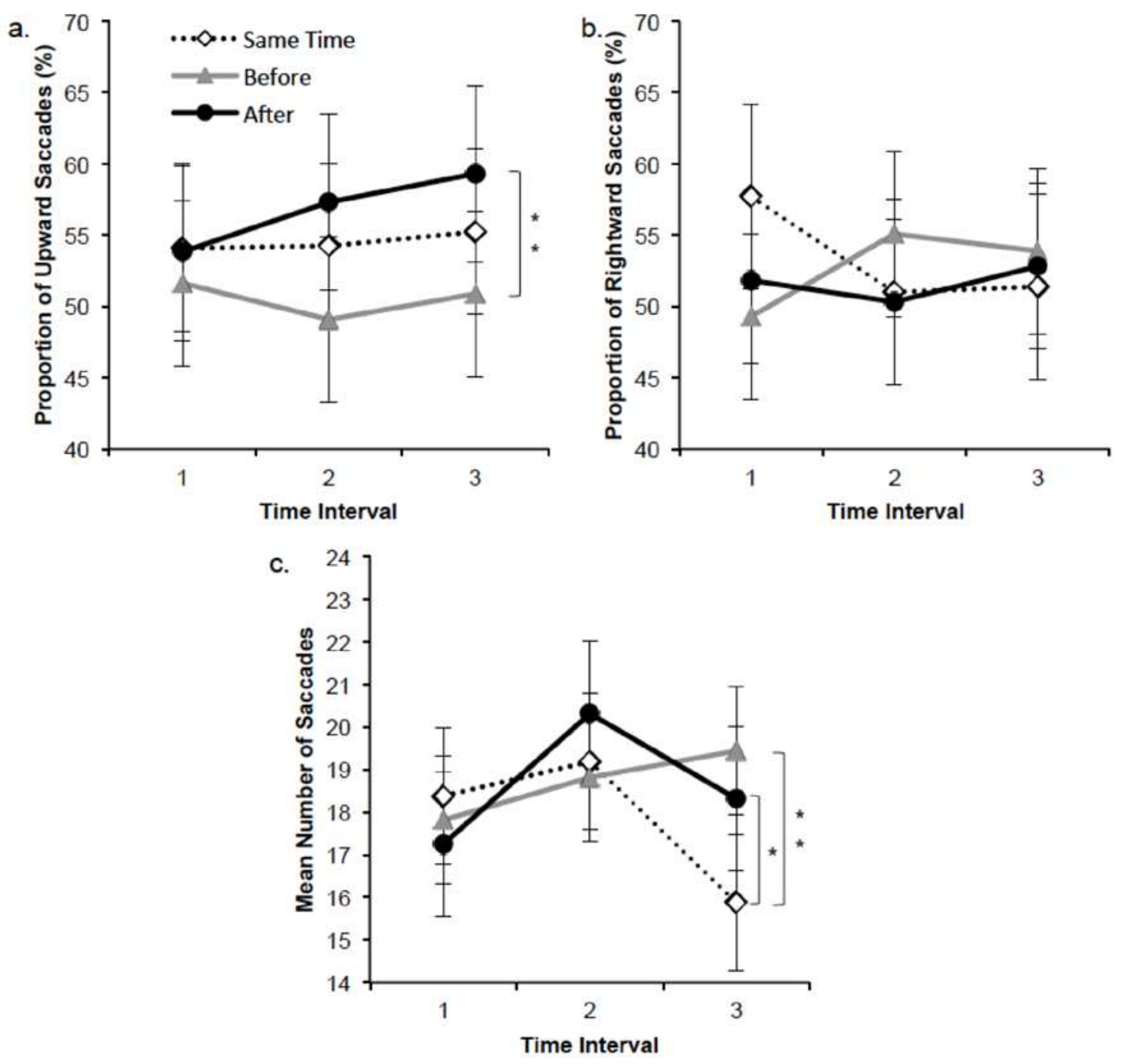

Figure 1. Mean proportion of upward (a) and rightward (b) saccades, and mean number of saccades (c), during 500-1500 ms (Interval 1), 1500-2500 ms (Interval 2), and 2500-3500 ms (Interval 3) after onset of the word expressing the temporal relation of the two events (before, after, same-time). ${ }^{*} p<.05, * * p<.01$. Error bars depict $95 \%$ within-subject confidence intervals. 
Our analysis showed that more upward saccades were made when processing future (after) than past (before) sentences. To further assess which saccades (e.g., straight upward directed saccades or diagonal directed saccades) drove the difference between the before and after sentences, we plotted the proportion of each saccade direction for the two conditions for the time intervals $2-3$ (showing the most pronounced differences, see Figure 1a). The plot

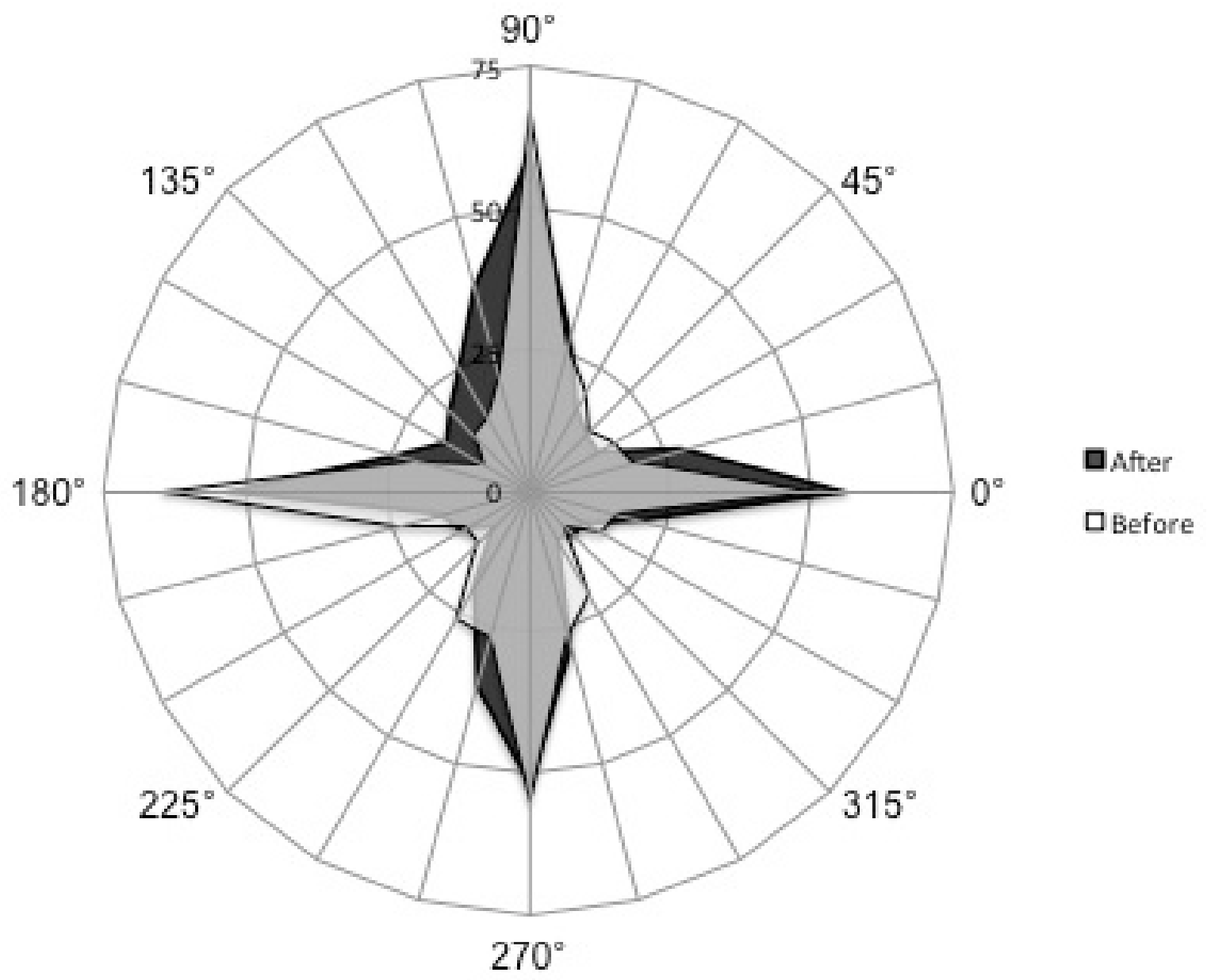

shows that the differences were mainly driven by saccades which_— besides a vertical component__also had a horizontal component, rather than by saccades which were just going straight up. 
Figure 2. Polar plot of the percentage of saccades in all directions during online processing of linguistically expressed time. White areas stand for saccades while processing the past (before), black areas for the future (after), and grey areas represent areas of past/future overlap.

Fewer fixations were made for same-time relations when compared to before and after relations. This suggests that different locations in time were mapped onto different locations in space. We further evaluated this hypothesis by analyzing more carefully the fixation patterns and the distribution characteristics of the different conditions. Figure 3 (left panel) shows the cumulative spatial distribution of fixations on the blank screen in each condition during the time intervals 2 and 3. Dark regions were attended more frequently, and the most attended regions are marked with solid lines. Visual inspection of these graphs reveals no obvious differences across conditions. As a more sensitive analysis, the right panel of Figure 3 shows the cumulative dwell time when the $\mathrm{x}$ and $\mathrm{y}$ coordinates are $\mathrm{z}$-standardized for each trial. The height of the 3D-graphs represents the bivariate kernel density estimation (see Huette, Winter, Matlock, \& Spivey, 2012 and Huette et al., 2014 for a similar analysis). Thus, not moving the eyes during a trial results in high density estimation of a single location, whereas moving the eyes during a trial leads to a wider distribution of density estimates and consequently to a lower local maxima and a less sharp peak (i.e., lower kurtosis) of the density estimation function. Interestingly, the same-time temporal relation shows the highest maximal density value ( 0.53 , compared to 0.37 for before and 0.39 for after temporal relations). Similarly, cumulative kurtosis along the y axis was highest in the same-time temporal relation (11.08, compared to 6.33 for before and 8.35 for after temporal relations). These results indicate that participants looked more often at the same position when processing events taking place at the same time than when events take place at different points in time. However, cumulative kurtosis along the $\mathrm{x}$ axis was similar across all conditions, and two-sample Kolmogorov-Smirnov tests along the $\mathrm{x}$ and $\mathrm{y}$ axis revealed no 
statistically significant difference between the three distributions (all $p$ s >0.05).

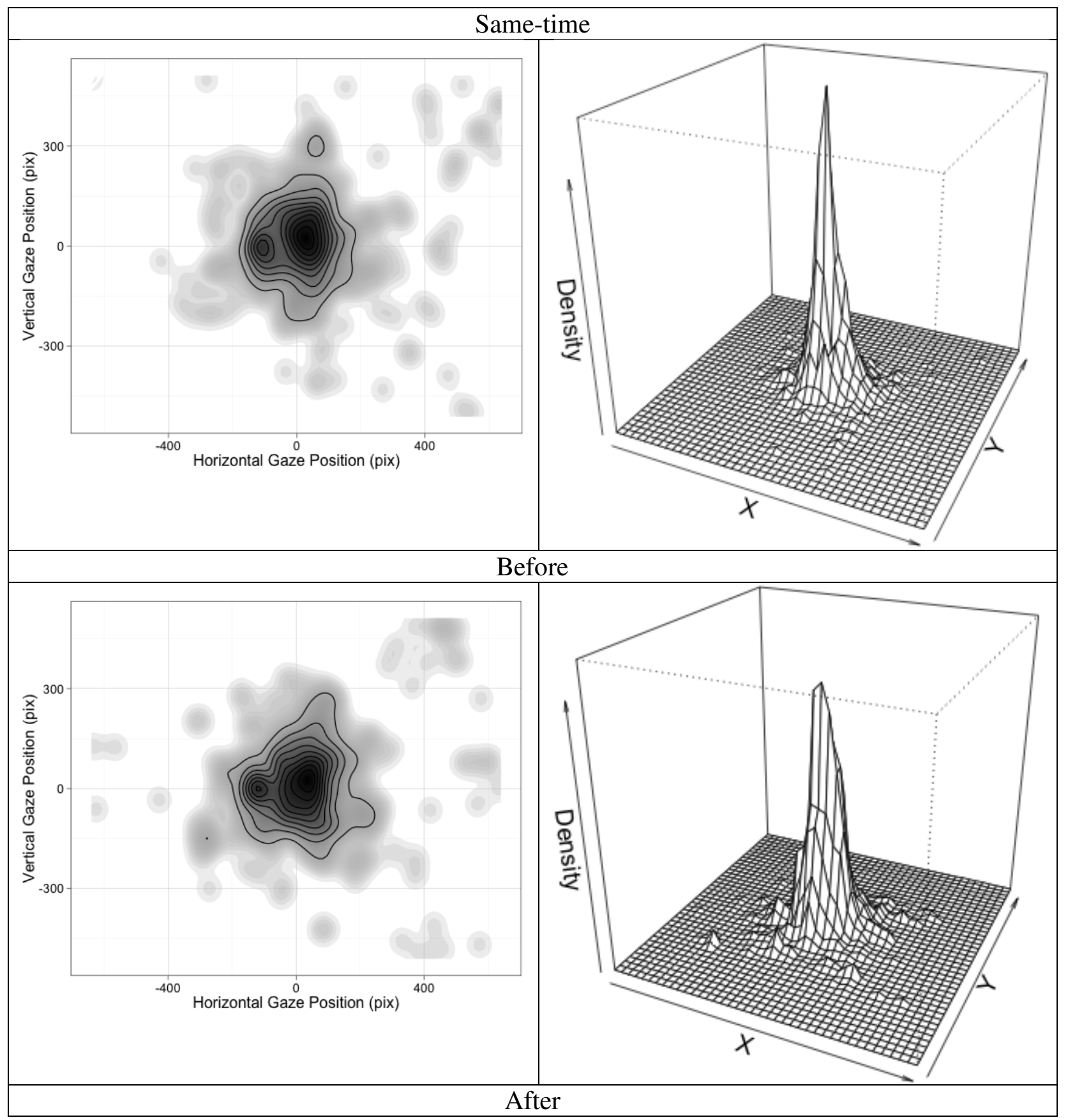




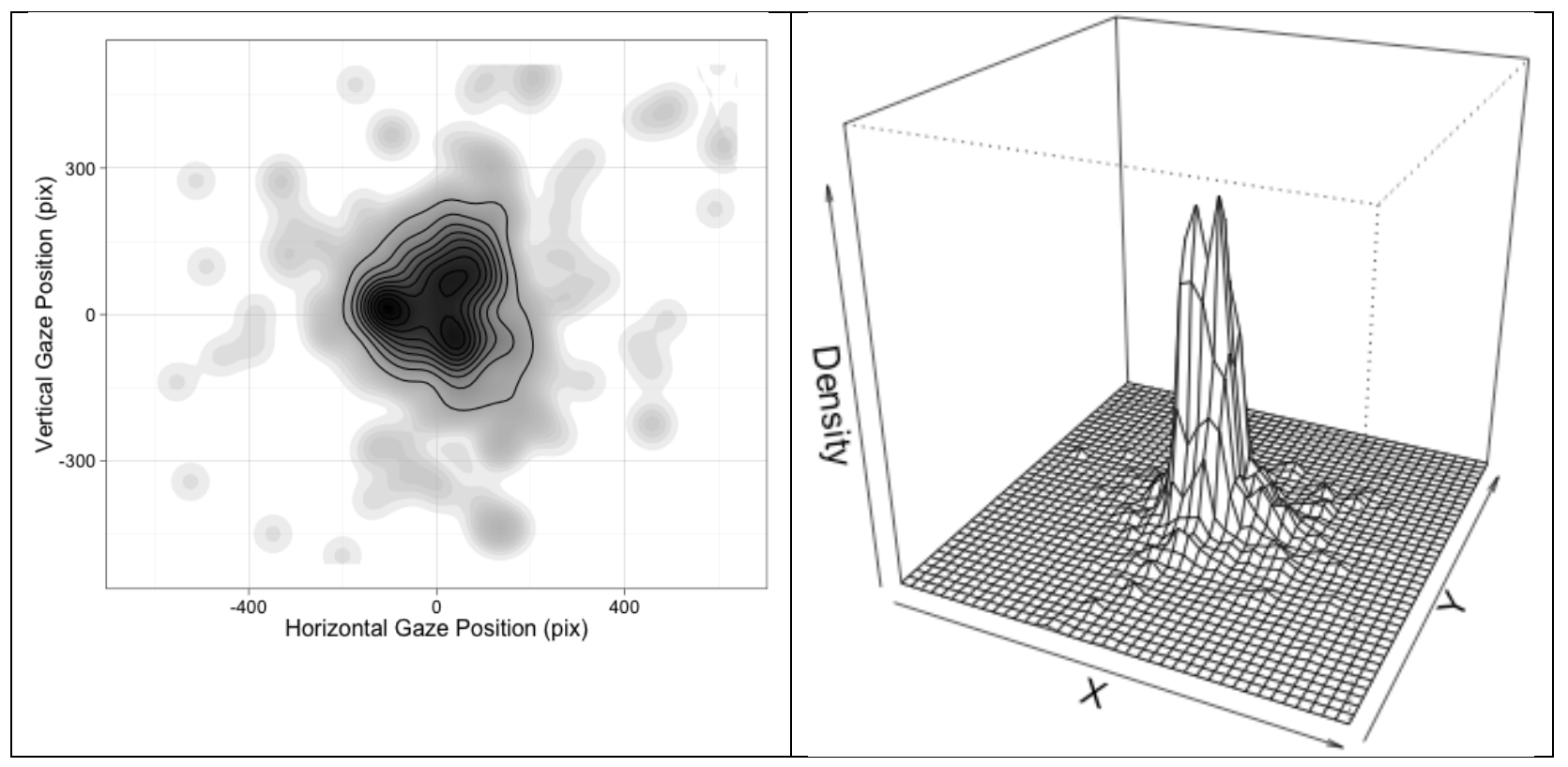

Figure 3. The left panel shows the cumulative distribution of fixations on the blank screen after the onset of the temporal relation words same-time, before, and after (for the time intervals 2 and 3). Dark regions were more attended, and the most attended regions are circled with solid lines. The right panel shows the cumulative dwell time on the blank screen when the $\mathrm{x}$ and $\mathrm{y}$ positions are $\mathrm{z}$-standardized for each trial. Fixating the same location during a trial results in a high peak of the density function; this is most pronounced for same-time relations.

\section{Discussion}

In this study we investigated whether the processing of different temporal relations in language (before, after, same-time) is systematically reflected in eye movements (i.e., eye movements that are not triggered in response to a perceptual event) on a blank screen. We found that significantly more upward saccades were made when processing after (future) than before (past) sentences. We interpret this finding in favor of our hypothesis that temporal processing evokes eye movements along the mental time line. As we mentally represent time, our mind's eye follows an upward_—and possibly forward_— progressing mental time line, and this is reflected in corresponding oculomotor correlates. To date, no 
evidence in any language or culture has been found for an exclusively upward-progressing mental time line (Bender \& Beller, 2014, p. 374). In contrast, there is evidence for an upward and forward progressing mental time line in a number of spoken and signed languages (Boyes Braem, 1992; Brien, 1992; Klima \& Bellugi, 1979; Lakoff \& Johnson, 1980a). Based on the paradigm we used in this study we were not able to measure the ocular depth dimension directly. ${ }^{3}$ Thus, we cannot conclude whether we identified eye movements along a vertical only or a vertical-sagittal mental time line. But in either case, our findings provide the first evidence that eye movements systematically reflect mental looking through time during language comprehension about time. The results suggest that the semantic processing of temporal relations is supported by the oculomotor system, structuring time in a metaphorically congruent manner into space.

The finding of a larger number of total saccades when processing before/after temporal relations than when processing same-time temporal relations lends additional support to our hypothesis that we mentally look along the mental time line. When processing two events occurring simultaneously, participants did not map them onto different spatial locations to the

3 There could be one way how our paradigm could detect sagittal mental projection into the screen. From binocular visual perception we know that our eyes converge more as they look at an object that is within proximal distance and diverge more as they look at an object that is more distally removed. This binocular phenomenon is commonly referred to as vergence. Thus, if the eyes mentally project more ("further") into the screen while processing the future, this could be reflected in a larger vergence angle for processing the future, as compared to the past. To investigate this possibility, we measured vergence for before (past) and after (future) relations. Vergence was assessed by computing the difference between the horizontal pupil position of the left and right eye for each sample in the raw data output. Since pupil position data were noisy during blinks and saccades, we only computed vergence during fixations. As we were only interested in possible vergence differences between the conditions that eventually developed from the onset of the critical time word, a baseline value was subtracted from each vergence value. Baseline was the individual vergence before the onset of the critical time word (assessed from averaging 5 samples before the onset). We first performed the same ANOVA as we performed for the proportion of saccades in our main analysis. This analysis revealed no significant vergence effects for before (past) versus after (future) relations (all ps > .153). We do not want to over-interpret this null finding because vergence typically occurs when the observer looks at an object that is either proximally or distally ahead of her or him (e.g., Alvarez, Semmlow, \& Pedrono, 2005). Such varying stimulus location was not given in our paradigm, as the looked-upon stimulus (the empty screen) always remained in one place. 
same extent as they did when two events occurred at two different points in time. However, while the standardized spatial distribution for same-time temporal relation indeed showed the highest kurtosis (indicating more fixations at the same position compared to before or after relations), the fixation differences were statistically not significant. Interestingly, Huette and colleagues (2014) found a much clearer influence of verb form manipulation on the spatial distribution of fixations. The weaker influence in our study could be due to the fact that we did not manipulate the degree of motion involved when simulating the described action. This then also shows a certain subtleness in our results_— that some of our results are subject to certain analytic conditions. Thus, when looking at the mean number of saccades (Fig. 1c), then significantly fewer saccades are made when cognitively processing two events that happen at the same time, compared to two events that happen at different points in time. When looking at cumulative dwell time (Figure 3, right panel), then the eyes fixate more on a single point when processing two events that happen at the same time, compared to two events that happen at different points. However, with the fixations_—unlike with the saccades_- this ocular focus on a single location no longer reaches significance. Thus, it remains an open question for future research how exactly the eyes are processing temporal simultaneity vs. temporal sequentiality.

While we found a difference in the proportion of upward saccades when processing future-related compared to past-related sentences, there was no significant difference between these two temporal categories and the present (same-time temporal relations). However, the proportion of upward saccades for the present was in between the values for future and past, suggesting a spatial representation of time of the format past-present-future. Noteworthy, our results point to potential asymmetries in this representation: the proportion of upward saccades for the present and the proportion of upward saccades for the future were more similar when compared to the proportion of upward saccades for the past. Thus it remains unclear whether our effect was driven by an increase in upward saccades when processing 
future, or an increase in downward saccades when processing past-related contents, or both.

Future studies on the mental time line could also incorporate a baseline in order to further assess possible asymmetries in the representation of time.

Given the well-established past-left and future-right association in Western culture (cf. Introduction), the absence of such an association in the current study is noteworthy. It suggests that mentally looking along a transversal left-to-right time line is not an indispensable requirement for comprehending and processing temporal information. This interpretation is also in line with the finding that the left-to-right mental time line is not automatically activated when processing temporal language (Ulrich \& Maienborn, 2010; Flumini \& Santiago, 2013). Our results support the recent claim that languagecomprehension tasks which do not require participants to make explicit temporal judgments only allow for using metaphor-driven mappings of concepts onto space (e.g., the future-front and future-up metaphors) (Sell \& Kaschak, 2011; Sell \& Kaschak, 2012). In contrast, tasks which require participants to make temporal judgments are more flexible in the use of space and, depending on the spatial reference imposed upon by the task settings, allow for compatibility effects that are not driven by metaphors, such as the past-left and future-right association (Sell \& Kaschak, 2011; Sell \& Kaschak, 2012; cf. also Santiago \& Lakens, $2015) .^{4}$

Interestingly, in another study of ours, which involved mental displacement into the personal future and past, we did find a past-left and future-right effect of eye movements-

4 In general, it is still unclear why judgment tasks seem to lead to back/front and left/right time effects while no-judgment tasks only show back/front effects. One possibility is that the sagittal time axis is the more basic time axis, as it is "going through the body" of the cognizer and that the left-right transversal time axis is a more "external time axis", since it can be used with or without projecting a self onto this time line (Hartmann et al., 2014; Núñez \& Cooperrider, 2013; cf. also Stocker, 2012). In case the transversal time line is more external, perhaps more cognitive resources (such as actively making a judgment) are needed to cognitively activate this time line. A recent study by Elkmeier, Alex-Ruf, Maienborn, and Ulrich (2015) has found that the back/front axis is more strongly associated with space than the left/right axis. This also speaks for the possibility that the sagittal axis is the more basic spatialized time line. 
or more precisely, a past-left/down and future-right/up effect (Hartmann et al., 2014). While participants mentally traveled one year into the past or future in this other study, the temporal differences between the events in the current study were much smaller (in the range of one day). A larger temporal distance might lead to more pronounced spatio-temporal associations (cf. Sell and Kaschak, 2011). Moreover, unlike in this study, our other study required participants to explicitly think about the future and the past for one minute. It is possible that left/right time effects in eye movements can only be found when participants are explicitly asked to create mental images of their past or future and not in small-time-scale (in the range of seconds) online language comprehension about time. In relation to this, it is also an interesting question what would have happened if we had instructed our participants to actively imagine the contents of the sentences (rather than passively listening to them). Perhaps an explicit active imagery component might also bring about a left-to-right effect even for processing generic (non-episodic) mental time (our temporal relations are one example of such generic time) even in a small time-scale context.

Another question for future research is whether processing the past could also activate the mental backspace (the space behind the body; e.g., Leuthard, Bächtold, \& Brugger, 2005; Viaud-Delmon, Brugger, \& Landis, 2007). It is possible that the past mental space is not only below the future mental space, but is additionally also conceptualized as being behind the body (below the future space and in the backspace). Sign languages often seem to project the past into the backspace_- they often signify the past with hand movements which are directed toward the backspace that is behind their shoulder (e.g., Brennan, 1983; Boyes Braem, 1992; Klima \& Bellugi, 1979). Thus, while it has been observed that the past can be conceptualized in relation to the backspace (signing space), for online language comprehension of time this question remains an open question.

Considering that the somewhat passive requirements of our paradigm on behalf of the participants_— to merely listen to sentences_—still evoked differences in vertical saccade 
direction, it stands to reason that the mental simulation of looking along an upward (and possibly also forward) progressing mental time line is activated automatically when cognitively processing time. While, in general, the question if mental simulation can be automatic is still a matter of debate (Kiefer \& Pulvermüller, 2012; Mahon \& Caramazza, 2009), there is at least some strong evidence that mental simulation of perceptual features of objects in space might be automatic (see Vukovic \& Williams, 2014). However, to date, strong support for the automaticity of mental simulation is still lacking in the realm of mental time. The transversal (left-right) mental time line has thus far not been found to be activated automatically (Ulrich \& Maienborn, 2010; Flumini \& Santiago, 2013), while studies investigating the same question for the sagittal (back-to-front) mental time line yielded inconclusive results (Sell \& Kaschak, 2011; Torralbo et al., 2006; Ulrich et al., 2012). Sell and Kaschak (2011) found such back-front activation during an atemporal discrimination task, which gives some evidence for automatic processing; but evidence for automaticity was only found for relatively large time shifts between the target events (e.g., mentally projecting one month into the past or future had an effect, while mentally projecting one day into the past or future did not). In contrast, Ulrich and colleagues found no sagittal effect whatsoever for temporal processing within a single sentence, and Torralbo and colleagues only found one in a language-production task. However, all language-comprehension findings that to date have investigated the automaticity of spatialized time (Sell \& Kaschak, 2011; Torralbo et al., 2006; Ulrich et al., 2012), have investigated body (hand and arm) motion through time and not, as we did, gaze direction through time (cf. also Hartmann et al., 2014; Stocker 2014a). It is possible that eye movements during the processing of temporal information are a more sensitive indicator for automatic spatiotemporal associations than manual responses. Future research is needed to address this question and to further clarify the conditions under which spatiotemporal frames of references are recruited automatically.

An alternative explanation for the oculomotor behavior which we show in this paper is 
a cohort effect (Marslen-Wilson, 1987). The (prepositional) word-part "vor" of our German temporal-adverb stimuli "vorher" does not only have a temporal (before), but also a spatial meaning (in front of). Similarly, "nach" of "nachher" does not only have a temporal (after), but also a spatial sense (to, as in going to some place). Thus, according to the cohort model, up to the moment when only the word-parts "vor" and "nach" were processed, their spatial meanings have also been activated as cohort competitors. One could therefore argue that the found effects have been driven by the spatial meanings of the cohort competitors. However, there is no logical reason why the spatial meaning in front of should be mapped lower in space than the spatial meaning to. Moreover, the spatial meaning of "nach" is (as is to in English) direction-unspecific, i.e., it can refer to any direction, depending on where the referred-to object is located in space. Thus, it is unlikely that such a direction-unspecific spatial meaning would yield such systematic direction-specific results as we have found.

Bringing the discussion to non-experimental fields of cognitive science, we can note that we have provided empirical (oculomotor) support for an assumption which can also be found in mathematical memory modeling (Brown, Neath, \& Chater, 2007) and cognitivelinguistic analysis (Stocker, 2012, 2014a, in press; cf. also Talmy, 2000, pp. 72-76, 86-87): for the assumption that we mentally look through time along a time line. Brown and colleagues (2007) have introduced a model of memory retrieval they call SIMPLE (scaleindependent memory, perception, and learning), a model which can explain and predict a vast amount of (free and serial recall) experimental memory data. This is how the SIMPLE model involves memory cognition along a mental time line:

... memory traces can be seen as located and individuated at least partly in terms of their position along a temporal continuum receding from the present into the past. This time line is logarithmically compressed, such that recent locations are more easily discriminable from one another than are more temporally distant locations (p. 541). 
Thus, SIMPLE assumes more spatial compression for more distant memory items than for more recent ones. This means that if, for instance, five memory items (A-B-C-D-E) are recalled, the spatial distance on the time line between A and B is assumed to be smaller than the one between $\mathrm{D}$ and $\mathrm{E}$, despite the fact that all memory items are assumed to have been presented with the same time interval between them. As it is assumed in SIMPLE, our current findings also suggest that temporal locations are "discriminable" along a mental time line-indeed, we have identified a possible physiological indicator which might be involved in discriminating these temporal points on the mental time line: correspondingly directed eye movements. Such a result opens up interesting venues for future research which is related to the SIMPLE model. In SIMPLE, more recent locations are more easily discriminable than certain more distant locations because more recent locations are spatially further apart from each other on the time line than more distant locations (which are spatially more compressed). This spatial compression for more distant memory items might for instance be oculomotorically reflected in smaller saccades between recalling memory item A and B than between recalling memory item $\mathrm{D}$ and $\mathrm{E}$ in a five-item serial recall task (A-B-C-D-E). As we in our study examined generic temporal relations (non-personal, non-episodic time), it is also worthwhile mentioning that SIMPLE has not only been successfully applied to episodic recall, but also to semantic recall, which also involves non-personal time (Kelley, Neath, \& Surprenant, 2012; Neath, 2010; Neath \& Brown, 2006; Neath \& Saint-Aubin, 2011).

Cognitive-linguistic work on mental time has also worked with the assumption that we mentally look along a time line (Stocker, 2012; Stocker, 2014a; Talmy, 2000). Such cognitive-linguistic work on time for instance involves testing certain hypotheses about linguistically expressed time by systematically contrasting acceptable and non-acceptable linguistic statements about time or time-related space. These overlaps between cognitivelinguistic findings and our current findings might encourage more oculomotor investigations 
on cognitive-linguistically derived postulates. Besides looking along a mental time line with the present moment as a starting point (which is the basic time concept that we have investigated in this current study), cognitive-linguistic work has also proposed other concepts in relation to mentally looking along a mental time line. To our knowledge, such concepts have hitherto not been investigated experimentally. One cognitive-linguistic example is mental looking at the time line in relation to two events where no present moment as a reference point is involved. This involves a form of temporal cognition called mental time watching and it can underlie expressions like "New Year's follows Christmas" (Stocker, 2012). Perhaps future work will also find systematic oculomotor reflections for these further cognitive-linguistic proposals of how we mentally look through time.

Our results extend a growing body of evidence showing that metaphors found in language manifest themselves in behavioral correlates. It has already been shown that eye movements follow a mental number line during random number generation (Loetscher et al., 2010) or partially during mental addition and subtraction (Hartmann et al., 2015) as well as along a mental time line during episodic memory and future thinking (Hartmann et al., 2014). Such results, as well as the results of the current study, show that the mind's eye does not only have the capacity to follow an outline of an imagined object within a spatial scene, but also has the capacity to follow an outline of a mentally invisible structure_- such as the mental number or time line.

Do eyes that follow such invisible spatial scaffoldings merely play an epiphenomenal role, or do they play a functional and facilitatory role in comprehending and processing abstract meaning? There is at least one recent study which points to the possibility that a spatialized time line might have functional status. Patients with spatial neglect (due to righthemisphere lesions) do not only neglect the left side of space, they also have difficulty with the retrieval of past events (left side of mental time line in Western and some other cultures) and no comparable difficulty with retrieval of future events (right side of mental time line in 
Western and some other cultures) (Saj, Fuhrman, Vuilleumier, \& Boroditsky, 2014).

Moreover, recent eyewitness memory studies have produced some first strong results that the use of a pictorial time line greatly assists in eliciting more specific memories and in greater accuracy of temporal sequence (Gosse \& Roberts, 2013; Hope, Mullis, \& Gabbert, 2013).

Another aspect that potentially points toward a functional role of spatializing time is the fact that every single culture or language in the world that has thus far been investigated shows some form of systematic spatial expression of time (Stocker, 2014b). Time is in many cultures indeed often mentally construed as a (more or less straight) line: from the past to the future, time has been found to flow from back to front or left to right in Western and many other cultures, from right to left in Hebrew and Arabic cultures, from top to bottom in Mandarin Chinese, from front to back in Aymara (i.e., the future is in the back and the past in the front), and even from east to west in Pormpuraaw, a remote Australian Aboriginal community. Time might also be represented in spatially bent ways (Yupno) or as two distinct spatial regions that are not linearly connected or as a circle (Yucatec Maya).

A promising future approach for investigating whether the functionality of spatialized time holds true cross-culturally would be to test "left-side-of-mental-time-line neglect" (in people with spatial neglect with right-hemisphere lesions) in Hebrew and Arabic cultures. If spatialized time were functional and at the same time showed cross-cultural variation, righthemisphere lesions should for instance lead to "past neglect" in Western culture (as investigated and found by Saj and colleagues, 2014), but should lead to "future neglect" in Hebrew and Arabic culture.

More direct investigation of the functionality of eyes looking along the mental time line could come from interference studies. One could use manipulation of eye movements in space/time-congruent and space/time-incongruent ways. In Western culture, looking up and right should facilitate the processing of future, and looking down and left should facilitate the processing of past-related information, while the opposite combinations should impair 
temporal processing. Again, this pattern should be reversed for Hebrew and Arabic cultures.

While the functional role of the spatialization of time remains to be determined by future studies, we have shown that observing eye movements is likely to "detect" invisible spatial scaffoldings which are involved in cognitively processing abstract meaning, even when the abstract meaning lacks an explicit spatial correlate.

\section{Acknowledgements}

Our thanks go out to the two anonymous reviewers for their extremely helpful and thoughtful remarks. We also thank Peter Brugger for discussing some of his data concerning neglect patients, Chris Bockisch for helpful advice for data analysis, and Michael Schwarzenbach (Phonogram Archive of the University of Zurich) for recording the audio sentence stimuli. K.S. was supported by the Cogito Foundation, Wollerau, Switzerland (R$135 / 12)$.

\section{References}

Altmann, G. (2004). Language-mediated eye movements in the absence of a visual world: The 'blank screen paradigm'. Cognition, 93(2), B79-B87.

Alvarez, T. L., Semmlow, J. L., \& Pedrono, C. (2005). Divergence eye movements are dependent on initial stimulus position. Vision Research, 45(14), 1847-1855.

Beech, J. R. (1980). Imaginal vs perceptual scanning of a visual representation. Perceptual and Motor Skills, 50(2), 367- 370.

Bender, A., \& Beller, S. (2014). Mapping spatial frames of reference onto time: A review of theoretical accounts and empirical findings. Cognition, 132(3), 342-382.

Bergen, B. K., \& Chan Lau, T. T. (2012). Writing direction affects how people map space onto time. Frontiers in Psychology, 3, 1-5.

Boroditsky, L. (2001). Does language shape thought?: Mandarin and English speakers' conceptions of time. Cognitive Psychology, 43(1), 1-22.

Boroditsky, L., \& Gaby, A. (2010). Remembrances of times east: Absolute spatial representations of time in an Australian aboriginal community. Psychological Science, 
21(11), 1635-1639.

Borst, G., \& Kosslyn, S. M. (2008). Visual mental imagery and visual perception: Structural equivalence revealed by scanning processes. Memory \& Cognition, 36(4), 849-862.

Boyes Braem, P. (1992). Einführung in die Gebärdensprache und ihre Erforschung [Introduction to sign language and sign language research] (2nd ed.). Hamburg: SignumVerlag.

Brandt, S. A., \& Stark, L. W. (1997). Spontaneous eye movements during visual imagery reflect the content of the visual scene. Journal of Cognitive Neuroscience, 9(1), 27-38.

Brennan, M. (1983). Marking time in British sign language. In J. Kyle \& B. Woll (Eds.), Language in sign: An international perpsective on sign language (pp. 10-31). London: Croom Helm.

Brien, D. (Ed.). (1992). Dictionary of British Sign Language (Compiled for the British Deaf Association by the Deaf Studies Research Unit, University of Durham). London, England: Faber and Faber.

Brown, G. D. A., Neath, I., \& Chater, N. (2007). A temporal ratio model of memory. Psychological Review, 114(3), 539-576.

Carreiras, M., \& Clifton, C., Jr. (2004). On the on-line study of language comprehension. In M. Carreiras \& C. Clifton, Jr. (Eds.), The on-line study of sentence comprehension: Eyetracking, ERP, and beyond (pp. 1-25). New York: Psychology Press.

Casasanto, D., \& Bottini, R. (2014). Mirror reading can reverse the flow of time. Journal of Experimental Psychology: General, 143(2), 473-479.

Casasanto, D., \& Jasmin, K. (2012). The hands of time: Temporal gestures in English speakers. Cognitive Linguistics, 23(4), 643-674.

Clark, H. H. (1973). Space, time, semantics, and the child. In T. E. Moore (Ed.), Cognitive development and the acquisition of language (pp. 27-63). New York: Academic Press.

Cooper, R. M. (1974). The control of eye fixation by the meaning of spoken language: A new methodology for the real-time investigation of speech perception, memory, and language processing. Cognitive Psychology, 6(1), 84-107.

Cooperrider, K., \& Núñez, R. E. (2009). Across time, across the body: Transversal temporal gestures. Gesture, 9(2), 181-206.

Dehaene, S., Bossini, S., \& Giraux, P. (1993). The mental representation of parity and number magnitude. Journal of Experimental Psychology: General, 122(3), 371-396.

Eikmeier, V., Alex-Ruf, S., Maienborn, C., \& Ulrich, R. (2015). How strongly linked are mental time and space along the left-right axis? Journal of Experimental Psychology: Learning, Memory, and Cognition. Advance online publication. http://dx.doi.org/10.1037/xlm0000129

Fischer, M. H., Castel, A. D., Dodd, M. D., \& Pratt, J. (2003). Perceiving numbers causes spatial shifts of attention. Nature Neuroscience, 6(6), 555-556.

Flumini, A., \& Santiago, J. (2013). Time (also) flies from left to right... if it is needed! In M. Knauff, M. Pauen, N. Sebanz, \& I. Wachsmuth (Eds.), Proceedings of the 35th Annual Conference of the Cognitive Science Society (pp. 2315- 2320). Austin, TX: Cognitive Science Society.

Gosse, L., \& Roberts, K. P (2014). Childrens use of a 'time line' to indicate when events occurred. Journal of Police and Criminal Psychology, 36-43. Advance online publication 28 Feb, 2013. DOI: 10.1007/s11896-013-9118-x 
Grant, E. R., \& Spivey, M. J. (2003). Eye movements and problem solving: Guiding attention guides thought. Psychological Science, 14, 462-466.

Hartmann, M., Grabherr, L., \& Mast, F. W. (2012). Moving along the mental number line: Interactions between whole-body motion and numerical cognition. Journal of Experimental Psychology: Human Perception and Performance, 38(6), 1416-1427.

Hartmann, M., Martarelli, C. S., Mast, F. W., \& Stocker, K. (2014). Eye movements during mental time travel follow a diagonal line. Consciousness and Cognition, 30, 201-209.

Hartmann, M., Mast, F. W., \& Fischer, M. H. (2015). Spatial biases during mental arithmetic: Evidence from eye movements on a blank screen. Frontiers in Psychology, 6, 12.

Hartmann, M., \& Mast, F. W. (2012). Moving along the mental time line influences the processing of future related words. Consciousness and Cognition, 21, 1558-1562.

Hope, L., Mullis, R., \& Gabbert, F. (2013). Who? What? When? Using a timeline technique to facilitate recall of a complex event. Journal of Applied Research in Memory and Cognition, 2, 20-24.

Hubbard, E. M., Piazza, M., Pinel, P., \& Dehaene, S. (2005). Interactions between number and space in parietal cortex. Nature Reviews Neuroscience, 6, 435- 448.

Huette, S., Winter, B., Matlock, T., Ardell, D. H., \& Spivey, M. (2014). Eye movements during listening reveal spontaneous grammatical processing. Frontiers in Psychology, 5, 410.

Huette, S., Winter, B., Matlock, T., \& Spivey, M. (2012). Processing motion implied in language: Eye-movement differences during aspect comprehension. Cognitive Processing, 13, 193-197.

Huettig, F., Rommers, J., \& Meyer, A. S. (2011). Using the visual world paradigm to study language processing: A review and critical evaluation. Acta Psychologica, 137(2), 151-171.

Johansson, R., Holsanova, J., \& Holmqvist, K. (2006). Pictures and spoken descriptions elicit similar eye movements during mental imagery, both in light and in complete darkness. Cognitive Science, 30(6), 1053-1079.

Johansson, R., \& Johansson, M. (2014). Look here, eye movements play a functional role in memory retrieval. Psychological Science, 25, 236-242.

Kaschak, M. P., Madden, C. J., Therriault, D. J., Yaxley, R. H., Aveyard, M., Blanchard, A. A., \& Zwaan, R. A. (2005). Perception of motion affects language processing. Cognition, 94(3), B79-B89.

Kelley, M. R., Neath, I., \& Surprenant, A. M. (2012). Three more semantic serial position functions and a SIMPLE explanation. Memory \& Cognition, 41, 600-610.

Kiefer, M., \& Pulvermüller, F. (2012). Conceptual representations in mind and brain: Theoretical developments, current evidence and future directions. Cortex, 48(7), 805-825.

Klima, E., \& Bellugi, U. (1979). The signs of language. Cambridge, MA: Harvard University Press.

Laeng, B., \& Teodorescu, D.-S. (2002). Eye scanpaths during visual imagery reenact those of perception of the same visual scene. Cognitive Science, 26, 207-231.

Lakoff, G., \& Johnson, M. (1980a). Conceptual metaphor in everyday language. The Journal of Philosophy, 77(8), 453- 486.

Lakoff, G., \& Johnson, M. (1980b). Metaphors we live by. Chicago: University of Chicago Press.

Lakoff, G., \& Johnson, M. (1999). Philosophy in the flesh. New York: Basic Books. 
Le Guen, O. (2012). An exploration in the domain of time: From Yucatec Maya time gestures to Yucatec Maya Sign Language time signs. In U. Zeshan \& C. de Vos (Eds.), Sign languages in village communities: Anthropological and linguistic insights (pp. 209-250). Berlin: De Gruyter.

Le Guen, O., \& Pool Balam, L. I. (2012). No metaphorical timeline in gesture and cognition among Yucatec Mayas. Frontiers in Psychology, 3, 271.

Leuthard, J., Bächtold, D., \& Brugger, P. (2005). Is "left" always where the thumb is right?: stimulus-response compatibilities as a function of posture and location of the responding hand. Cognitive and Behavioral Neurology, 18(3), 173-178.

Lindsay, S., Scheepers, C., \& Kamide, Y. (2013). To dash or to dawdle: Verb-associated speed of motion influences eye movements during spoken sentence comprehension. PloS one, 8(6), e67187.

Loetscher, T., Bockisch, C. J., Nicholls, M. E. R., \& Brugger, P. (2010). Eye position predicts what number you have in mind. Current Biology, 20(6), R264-R264.

Lourenco, S. F., \& Longo, M. R. (2010). General magnitude representation in human infants. Psychological Science, 21(6), 873-881.

Mahon, B. Z., \& Caramazza, A. (2009). Concepts and categories: A cognitive neuropsychological perspective. Annual Review of Psychology, 60, 27-51.

Marslen-Wilson, W. D. (1987). Functional parallelism in spoken word-recognition. Cognition, 25(1), 71-102.

Martarelli, C. S., \& Mast, F. W. (2011). Preschool children's eye-movements during pictorial recall. British Journal of Developmental Psychology, 29, 425- 436.

Miles, L. K., Betka, E., Pendry, L. F., \& Macrae, C. N. (2010a). Mapping temporal constructs: Actions reveal that time is a place. The Quarterly Journal of Experimental Psychology, 63(11), 2113-2119.

Miles, L. K., Nind, L. K., \& Macrae, C. N. (2010b). Moving through time. Psychological Science, 21(2), 222-223.

Miles, L. K., Tan, L., Noble, G. D., Lumsden, J., \& Macrae, C. N. (2011). Can a mind have two time lines? Exploring space--time mapping in Mandarin and English speakers. Psychonomic Bulletin \& Review, 18(3), 598-604.

Mishra, R. K., \& Singh, N. (2010). Online fictive motion understanding: An eye-movement study with Hindi. Metaphor and Symbol, 25(3), 144-144.

Neath, I. (2010). Evidence for similar principles in episodic and semantic memory: The presidential serial position function. Memory \& Cognition, 38(5), 659-666.

Neath, I., \& Brown, G. D. A. (2006). SIMPLE: Further applications of a local distinctiveness model of memory. In B. H. Ross (Ed.), Psychology of learning and motivation (Vol. 46, pp. 201-243). San Diego, CA: Academic Press.

Neath, I., \& Saint-Aubin, J. (2011). Further evidence that similar principles govern recall from episodic and semantic memory: The Canadian prime ministerial serial position function. Canadian Journal of Experimental Psychology/Revue Canadienne de Psychologie Expérimentale, 65, 77-83.

Núñez, R., \& Cooperrider, K. (2013). The tangle of space and time in human cognition. Trends in Cognitive Sciences, 17(5), 220-229.

Núñez, R., Cooperrider, K., Doan, D., \& Wassmann, J. (2012). Contours of time: Topographic construals of past, present, and future in the Yupno valley of Papua New 
Guinea. Cognition, 124, 25-35.

Núñez, R. E., \& Sweetser, E. (2006). With the future behind them: Convergent evidence from Aymara language and gesture in the crosslinguistic comparison of spatial construals of time. Cognitive Science, 30(3), 401-450.

Ooi, T. L., Wu, B., \& He, Z. J. (2001). Distance determined by the angular declination below the horizon. Nature, 414(6860), 197-200.

Richardson, D., \& Matlock, T. (2007). The integration of figurative language and static depictions: An eye movement study of fictive motion. Cognition, 102, 129-138.

Richardson, D. C., \& Spivey, M. J. (2000). Representation, space and Hollywood Squares: Looking at things that aren't there anymore. Cognition, 76(3), 269-295.

Saj, A., Fuhrman, O., Vuilleumier, P., \& Boroditsky, L. (2014). Patients with left spatial neglect also neglect the "left side" of time. Psychological Science, 25(1), 207-214.

Santiago, J., \& Lakens, D. (2015). Can conceptual congruency effects between number, time, and space be accounted for by polarity correspondence? Acta Psychologica, 156, 179-191.

Santiago, J., Lupáñez, J., Pérez, E., \& Funes, M. J. (2007). Time (also) flies from left to right. Psychonomic Bulletin \& Review, 14(3), 512-516.

Sell, A. J., \& Kaschak, M. P. (2011). Processing time shifts affects the execution of motor responses. Brain and Language, 117(1), 39-44.

Sell, A. J., \& Kaschak, M. P. (2012). The comprehension of sentences involving quantity information affects responses on the up-down axis. Psychonomic Bulletin \& Review, 19(4), 708-714.

Sinha, C., Sinha, V. D. S., Zinken, J., \& Sampaio, W. (2011). When time is not space: The social and linguistic construction of time intervals and temporal event relations in an Amazonian culture. Language and Cognition, 3(1), 137-169.

Speed, L. J., \& Vigliocco, G. (2014). Eye movements reveal the dynamic simulation of speed in language. Cognitive Science, 38(2), 367-382.

Spivey, M. J., \& Geng, J. J. (2001). Oculomotor mechanisms activated by imagery and memory: Eye movements to absent objects. Psychological Research, 65, 235-241.

Stocker, K. (2012). The time machine in our mind. Cognitive Science, 36(3), 385-420.

Stocker, K. (2014a). Mental perspective in multiple-event memory and foresight. Journal of Cognitive Science, 15(1), 57-95.

Stocker, K. (2014b). The theory of cognitive spacetime. Metaphor and Symbol, 29(2), 71-93.

Stocker, K. (in press). Toward an embodied cognitive semantics. Cognitive Semantics [to appear in 2015. Vol 1, Issue 2].

Talmy, L. (1996). Fictive motion in language and "ception'. In P. Bloom, M. A. Peterson, \& M. F. Garrett (Eds.), Language and space (pp. 211-276). Cambridge, MA: MIT Press.

Talmy, L. (2000). Toward a cognitive semantics. Vol. 1: Concept structuring systems. Cambridge, MA: MIT Press.

Tanenhaus, M. K., Spivey-Knowlton, M. J., Eberhard, K. M., \& Sedivy, J. C. (1995). Integration of visual and linguistic information in spoken language comprehension. Science, 268(5217), 1632-1634.

Torralbo, A., Santiago, J., \& Lupiañez, J. (2006). Flexible conceptual projection of time onto spatial frames of reference. Cognitive Science, 30, 745-757. 
Tversky, B., Kugelmass, S., \& Winter, A. (1991). Cross-cultural and developmental trends in graphic productions. Cognitive Psychology, 23, 515-557.

Ulrich, R., Eikmeier, V., de la Vega, I., Ruiz Fernández, S., Alex-Ruf, S., \& Maienborn, C. (2012). With the past behind and the future ahead: Back-to-front representation of past and future sentences. Memory \& Cognition, 40, 483-495.

Ulrich, R., \& Maienborn, C. (2010). Left--right coding of past and future in language: The mental timeline during sentence processing. Cognition, 117(2), 126-138.

Van Gompel, R. P. G., Fischer, M. H., Murray, W., \& Hill, R. L. (2007). Eye movements: A window on mind and brain. Oxford: Elsevier.

Viaud-Delmon, I., Brugger, P., \& Landis, T. (2007). Hemineglect: Take a look at the back space. Annals of Neurology, 62(4), 418-422.

Vukovic, N., \& Williams, J. N. (2014). Automatic perceptual simulation of first language meanings during second language sentence processing in bilinguals. Acta Psychologica, 145, 98-103.

Yang, Z., \& Purves, D. (2003). A statistical explanation of visual space. Nature Neuroscience, 6(6), 632- 640. 